\title{
Medicinal value of asiaticoside for Alzheimer's disease as assessed using single-molecule- detection fluorescence correlation spectroscopy, laser-scanning microscopy, transmission electron microscopy, and in silico docking
}

Shahdat Hossain ${ }^{1,2}$, Michio Hashimoto ${ }^{1 *}$, Masanori Katakura ${ }^{1}$, Abdullah Al Mamun ${ }^{1}$ and Osamu Shido ${ }^{1}$

\begin{abstract}
Background: Identifying agents that inhibit amyloid beta peptide $(A \beta)$ aggregation is the ultimate goal for slowing Alzheimer's disease (AD) progression. This study investigated whether the glycoside asiaticoside inhibits $A \beta_{1-42}$ fibrillation in vitro.

Methods: Fluorescence correlation spectroscopy (FCS), evaluating the Brownian diffusion times of moving particles in a small confocal volume at the single-molecule level, was used. If asiaticoside inhibits early $A \beta_{1-42}$ fibrillation steps, more Aßs would remain free and rapidly diffuse in the confocal volume. In contrast, "weaker or no inhibition" permits a greater number of $A \beta s$ to polymerize into oligomers, leading to fibers and gives rise to slow diffusion times in the solution. Trace amounts of 5-carboxytetramethylrhodamine (TAMRA)-labeled $A \beta_{1-42}$ in the presence of excess unlabeled $A \beta_{1-42}(10 \mu M)$ was used as a fluorescent probe. Steady-state and kinetic-Thioflavin T (ThT) fluorospectroscopy, laser-scanning fluorescence microscopy (LSM), and transmission electron microscopy (TEM) were also used to monitor fibrillation. Binding of asiaticoside with $A \beta_{1-42}$ at the atomic level was computationally examined using the Molegro Virtual Docker and PatchDock.

Results: With $1 \mathrm{~h}$ of incubation time for aggregation, FCS data analysis revealed that the diffusion time of TAMRA-A $\beta_{1-42}$ was $208 \pm 4 \mu \mathrm{s}$, which decreased to $164 \pm 8.0 \mu \mathrm{s}$ in the presence of asiaticoside, clearly indicating that asiaticoside inhibited the early stages $A \beta_{1-42}$ of fibrillation, leaving more free $A \beta s$ in the solution and permitting their rapid diffusion in the confocal volume. The inhibitory effects were also evidenced by reduced fiber formation as assessed by steady-state and kinetic ThT fluorospectroscopy, LSM, and TEM. Asiaticoside elongated the lag phase of $A \beta_{1-42}$ fibrillation, indicating the formation of smaller amyloid species were impaired in the presence of asiaticoside. Molecular docking revealed that asiaticoside binds with amyloid intra- and inter-molecular amino acid residues, which are responsible for $\beta$-sheet formation and longitudinal extension of fibrils.
\end{abstract}

Conclusion: Finally, asiaticoside prevents amyloidogenesis that precedes neurodegeneration in patients with Alzheimer's disease.

Keywords: Asiaticoside, Amyloid fibrillation, Fluorescence correlation spectroscopy, Neurotoxicity, Molecular Docking

\footnotetext{
*Correspondence: michio1@med.shimane-u.ac.jp

'Department of Environmental Physiology, Shimane University Faculty of Medicine, Izumo 693-8501, Japan

Full list of author information is available at the end of the article
} 


\section{Background}

Alzheimer's disease $(\mathrm{AD})$ is pathologically characterized by the fibrillar deposition of amyloid beta peptides $(A \beta)$ in the brain [1]. $A \beta_{1-40}$ prevails in the cerebrospinal fluid of patients with $\mathrm{AD}$ [2]. However, $\mathrm{A} \beta_{1-42}$ deposition prevails in the brains of patients with $\mathrm{AD}$ [3], thereby implicating its involvement in the initiation of fibrillation. However, the mechanisms of $A \beta$ fibrillation remain elusive. Several key events are considered to be crucial, including the mechanism by which $A \beta_{1-42}$ abandons its random coil-alpha helix conformation and adapts a $\beta$-sheet conformation, leading to oligomerization and finally matured fiber formation. These $\alpha$-to- $\beta$ conformational transitions are crucial for understanding the mechanisms of fiber formation and inhibition by agents capable of inhibiting the fibrillation process.

Asiaticoside, a highly polyphenolic compound, is one of the major triterpene glycosides in Centella asiatica (CA). CA is an extremely important medicinal herb used in Java and other Indonesian islands, China [4], and other Asian countries [5], and is also becoming popular in Western countries [6]. Asiaticoside retains inherent properties to combat oxidative species, which are frequently observed in patients with $\mathrm{AD}$-associated memory impairments $[7,8]$. CA increases intelligence and memory [9]. Asiaticoside also has been reported to protect against $A \beta$-induced neurotoxicity [10]. More importantly, asiaticoside has been patented as a dementia-treating agent and cognitive enhancer [11]. However, the mechanisms of action of asiaticoside have remained largely unknown.

In this study, we primarily used fluorescence correlation spectroscopy (FCS) to examine whether asiaticoside inhibits $A \beta_{1-42}$ fibrillation and its mechanism. FCS is a new method that can detect molecular motion at the nanomolar level in small sample volumes. This technique has recently attracted interest for investigating the molecular interactions of proteins $[12,13]$. FCS allows real-time monitoring of protein-protein interactions in a reaction solution without separation of the free and bound forms $[14,15]$. For example, FCS can be successfully used in an aggregating system using trace amounts of 5-carboxytetramethylrhodamine (TAMRA)labeled $A \beta_{1-42}$ in the presence of a large excess of unlabeled $A \beta_{1-42}$ in a solution [16]. During aggregation, the fluorescent species will remain constant (because of the large excess of unlabeled molecules), and the diffusion time will gradually increase. Fluctuations in the fluorescence signal in a detection volume of approximately $1 \mathrm{fl}$ (femtoliter) are analyzed using an autocorrelation function, revealing information about the diffusion properties of the fluorescent complexes; larger average complex sizes are associated with longer diffusion times. Changes in the average diffusion time reflect changes in the complex size and/or the ratio of free fluorescently labeled molecules in the complexes.
In addition, steady-state and kinetic thioflavin $\mathrm{T}$ (ThT) fluorospectroscopy, transmission electron microscopy (TEM), and laser-scanning fluorescence microscopy (LSM) were used to elucidate the mechanism of asiaticoside-induced inhibition of $\mathrm{A} \beta_{1-42}$ fibrillation. In the field of molecular modeling, docking is a method that predicts the preferred orientation of one molecule to a second when bound to each other as a stable complex [17]. At present, the use of computers to predict the binding of small molecules to known target protein structures has been an important component in the drug discovery process $[18,19]$. However, there is no conclusive report regarding whether the asiaticoside docks onto $A \beta_{1-42}$, and if so, the amino acid specificity with which it binds as ligand to inhibit amyloid aggregation is unclear. We, therefore, investigated whether asiaticoside binds with amyloidogenic hot spots, i.e., the amino acid residues involved in $\beta$-aggregation, which may further support the use of asiaticoside as an amyloidogenesis-inhibitory agent.

\section{Methods \\ Materials}

$\mathrm{A} \beta_{1-42}$ (human, 1-42) was purchased from the Peptide Institute (Osaka, Japan). Asiaticoside was purchased from Sigma-Aldrich. The reference dye 5-carboxytetra methylrhodamine (TAMRA) was purchased from Olympus America Inc, whereas TAMRA-A $\beta_{1-42}$ was obtained from ANASPEC Inc. CA. Other chemicals were of analytical grade. Uranyl acetate was obtained from BDH. All experiments were carried out with the approval of an appropriate ethics committee of Shimane University compiled from the Guidelines for Animal Experimentation of the Japanese Association for Laboratory Animal Science.

\section{Preparation of asiaticoside, TAMRA-A $\beta_{1-42}$, and unlabeled $A \beta_{1-42}$}

Asiaticoside was dissolved in ethanol, diluted, $\mathrm{N}_{2}$-dried to remove ethanol, and then mixed with assembly buffer to have final concentrations of 5, 10 and $20 \mu \mathrm{M}$. TAMRA-A $\beta_{1-42}$ and unlabeled $A \beta_{1-42}$ were dissolved in hexafluoroisopropanol (HFIP), aliquoted, and stored at $-80^{\circ} \mathrm{C}$ until use. HFIP was also blown with $\mathrm{N}_{2}$ prior to the use in fibrillation assay.

\section{Fluorescence Correlation Spectroscopy (FCS) Theory}

In an FCS experiment, fluctuations of the fluorescence $\delta \mathrm{F}(\mathrm{t})$ around the average fluorescence $<\mathrm{F}(\mathrm{t})>$ are measured, yielding information on molecular processes or motions. The fluctuations of the fluorescence signal, $\delta \mathrm{F}(\mathrm{t})$, stem from changes in either the number of fluorescent particles or the fluorescence quantum yield of the particles in the open probe volume, which is defined by the confocal volume of a 
tightly focused laser beam. To analyze these fluctuations, the autocorrelation function $\mathrm{G}(\mathrm{\tau})$ of the fluorescence intensity is calculated using the following equation:

$$
\mathrm{G}(\mathrm{\tau})=\frac{\langle\delta \mathrm{F}(\mathrm{t}) \delta \mathrm{F}(\mathrm{t}+\mathrm{\tau})\rangle}{\langle\mathrm{F}(\mathrm{t})\rangle^{2}}
$$

where $\tau$ is the correlation time. $F(t)$ signifies the detected fluorescence intensity, where $\delta \mathrm{F}(\mathrm{t})$ is the variable, fluctuating part and $\langle\mathrm{F}(\mathrm{t})\rangle$ denotes the mean. The angular brackets indicate a time average, $<\delta \mathrm{F}(\mathrm{t}) \delta \mathrm{F}(\mathrm{t}+\tau)>$ is the average product of a fluctuations amplitude at time $\mathrm{t}$ and a later time $(\tau),(\mathbf{t}+\tau)$. F denotes the fluorescence signal as a function of time (see Figure 1).

FCS measurements were performed on a Fluoro Point Light system (Olympus, Tokyo, Japan) at room temperature using the onboard 543-nm helium-neon laser at a power of $100 \mu \mathrm{W}$ for excitation, as previously described [20]. TAMRA-A $\beta_{1-42} /$ unlabeled $A \beta_{1-42}$ dissolved in HFIP was blown with $\mathrm{N}_{2}$ gas and re-dissolved in the assembly buffer with or without asiaticoside. Free TAMRA (rhodamine) was used as a reference dye. The final concentrations of TAMRA-A $\beta_{1-42}$ and unlabeled-A $\beta_{1-42}$ were $5 \mathrm{nM}$ and 10 $\mu \mathrm{M}$, respectively. The measurements were performed in a sample volume of $50 \mu \mathrm{l}$ in the 384-well glass-bottomed microplate. The samples were sequentially and automatically loaded into the device. FCS measurements were conducted at 0 and $1 \mathrm{~h}$ with a data acquisition time of $10 \mathrm{~s}$ per measurement, and measurements were repeated five times per sample.

\section{Steady-state $A \beta_{1-42}$ fibrillation analysis using ThT fluorospectroscopy}

$\mathrm{A} \beta_{1-42}$ fibrillation was induced as previously described $[8,21]$. After blowing HFIP from the $A \beta_{1-42}$ stock aliquot, the dried peptide $(50 \mu \mathrm{M})$ was suspended in desired volume of assembly buffer (100 $\mu$ l of $50-\mathrm{mM}$ Tris- $\mathrm{HCl}$ buffer, $\mathrm{pH}$ 7.4, containing $100 \mathrm{mM} \mathrm{NaCl}$ and $0.01 \%$ sodium azide) with or without asiaticoside (final concentrations of asiaticoside were $0,5,10$, and $20 \mu \mathrm{M}$ ). The reaction mixture was taken in oil-free PCR tubes (Takara Shuzo, Otsu, Japan) flushed with of $\mathrm{N}_{2}$ gas to obviate any effect of atmospheric oxygen, and incubated at $37^{\circ} \mathrm{C}$ for $24 \mathrm{~h}$ into a DNA thermal cycler (PJ480; Perkin Elmer Cetus, Emeryville, CA). After $24 \mathrm{~h}$, the incubation was stopped by placing the tubes on ice and 40- $\mu$ l aliquots from each tube were mixed with 210 $\mu \mathrm{l}$ of $5-\mu \mathrm{M}$ ThT in $50 \mathrm{mM}$ glycine- $\mathrm{NaOH}$ buffer $(\mathrm{pH} 8.5$ ) and subjected to fluorospectroscopy (Hitachi F-2500 fluorescence spectrophotometer) at excitation and emission wavelengths of 448 and $487 \mathrm{~nm}$, respectively.

Effects of asiaticoside on the kinetics of $A \beta_{1-42}$ fibrillation The effect of asiaticoside on the kinetics of fibrillation was evaluated by using $10 \mu \mathrm{M}$ of $\mathrm{A} \beta_{1-42}$. Briefly, the reaction mixture containing $1.0 \mathrm{ml}$ of $50 \mathrm{mM}$ Tris- $\mathrm{HCl}$ buffer, $\mathrm{pH}$ $7.4,100 \mathrm{mM} \mathrm{NaCl}, 0.01 \%$ sodium azide, and the desired amount of $A \beta_{1-42}$ with or without asiaticoside $(20 \mu \mathrm{M})$ was taken into Eppendorf tubes and incubated at $37^{\circ} \mathrm{C}$. At desired time intervals, a $40-\mu \mathrm{l}$ of the peptide mixture was gently removed and added to $210 \mu \mathrm{l}$ of $5 \mu \mathrm{M}$ ThT in 50 $\mathrm{mM}$ glycine- $\mathrm{NaOH}$ buffer ( $\mathrm{pH} 8.5$ ) for fluorescence assay.

\section{$A \beta_{1-42}$ fibrillation analysis using Laser Scanning Microscopy (LSM)}

A $2.5-\mu \mathrm{l}$ aliquot of the fibrillated $A \beta_{1-42}$ peptide $(50 \mu \mathrm{M})$ sample from the ThT-A $\beta_{1-42}$ assay with or without asiaticoside $(20 \mu \mathrm{M})$ was diluted 2-fold with $5 \mu \mathrm{M}$ ThT in $50 \mathrm{mM}$ glycine- $\mathrm{NaOH}$ buffer $(\mathrm{pH} 8.5)$, transferred onto slides, and photographed using the confocal laser microscope system (CLSM FV300; Olympus). The fibrillation of $5 \mathrm{nM}$ TAMRA-A $\beta_{1-42}+20 \mu \mathrm{M}$ unlabeled $\mathrm{A} \beta_{1-42}$ with or without $20 \mu \mathrm{M}$ asiaticoside was also conducted. Aggregates

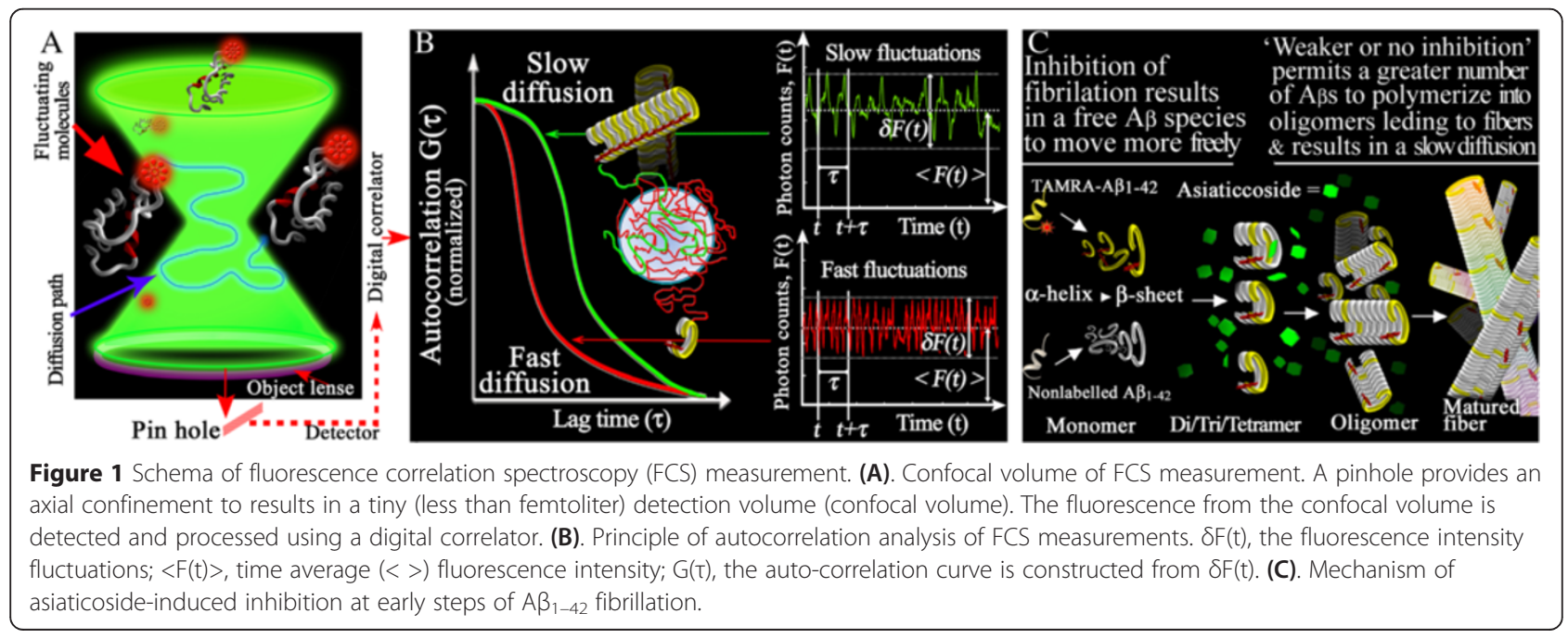


of TAMRA-A $\beta_{1-42}$ were directly visualized under the microscope at an excitation wavelength of $542 \mathrm{~nm}$.

\section{$A \beta_{1-42}$ fibrillation analysis using TEM}

Fibrillation of $A \beta_{1-42}(50 \mu \mathrm{M})$ was induced as described for ThT fluorospectroscopy. A 4- $\mu \mathrm{l}$ aliquot was used for electron microscopy. In brief, a droplet of the reaction mixture was spread onto carbon-coated grids, negatively stained with $1 \%$ uranyl acetate (pH 7.0), and examined under a Hitachi H-7000 electron microscope with an acceleration voltage of $75 \mathrm{kV}$.

\section{Docking study: Preparation of docking materials (ligand and receptor)}

The canonical SMILES string of asiaticoside (PubChem: CID 108062; Figure 2) was submitted to Marvin 5.7.0 [22] to generate the 3D structure of this ligand molecule. The 3D structure of asiaticoside was subsequently energy-minimized and converted to the Protein Data Bank (PDB) file format using the Molegro Virtual Docker (MVD) [23]. A $\beta_{1-42}$ (PDB ID: 2BEG) was downloaded from the Protein Data Bank as a receptor for asiaticoside docking. 2BEG is a 3D NMR structure of $A \beta_{1-42}$, consisting of a homopentamer $(A, B, C, D$, and E). Each monomer of the 2 BEG pentamer comprises 10 coordinate models [24].

\section{Dimer formation}

At least two amyloid molecules are required to achieve the repeating structure of a protofilament fibril; therefore, the coordinates of model 1 of A monomer (A1) and model 1 of $\mathrm{B}$ monomer (B1) were split from the composite 2BEG PDB file by MVD. Subsequently, the monomer (A1)-monomer (B1) dimer (A1-B1) was generated by feeding the A1 and B1 monomers to RosettaDock [25], which decoys 1000 independent simulations and gives the $\mathrm{A} 1-\mathrm{B} 1$ dimer on the basis of energy minimization.

\section{Analysis of the unstructured and aggregation-prone regions of $A \beta_{1-42}$}

The FASTA format of the primary amino acid sequence of $A \beta_{1-42}$ (>seq DAEFRHDSGYEVHHQKLVFFAEDVGSNK GAIIGLMVGGVVIA) was tested by ANCHOR [26] to elucidate the intrinsically unstructured region of this peptide. In addition, we used three state-of-the-art sequencebased computational methods, namely FoldAmyloid [27], AGGRESCAN [28], and ProA [29], to predict the amyloidogenic regions and aggregation-prone amino acid residues from $A \beta_{1-42}$ peptide chains.

\section{Computational analysis of binding sites of $A \beta_{1-42}$ monomers and dimers}

The binding sites or pockets of the A1-monomer and A1-B1 dimer were determined by GHECOM, which detects grid-based pockets/cavities on the surface of the protein [30]. The program produces a graph of residue-based pocketness. The presence of binding sites was also cross-checked using Q-SiteFinder [31], which uses the interaction energy between the protein and a simple van der Waals probe to locate energetically favorable binding sites.

\section{Intersurface interaction site analysis of the A1-B1 dimer} Protein-protein, i.e., monomer-monomer A1-B1 dimer, intersurface interaction sites were analyzed by feeding the A1-B1 dimer to the cons-PPISP server [32], which predicts the residues that likely form the binding site for neighbor proteins. To further validate the presence of intersurface hot points on the dimer interface binding contacts, the dimer was fed to hot point prediction servers, including KFC2 [33,34].

Docking simulation of asiaticoside onto $A \beta_{1-42}$ monomer (A1) and dimer (A1-B1)

The molecular docking simulations were performed using the Molegro Virtual Docker (MVD) [23] and PatchDock [35].

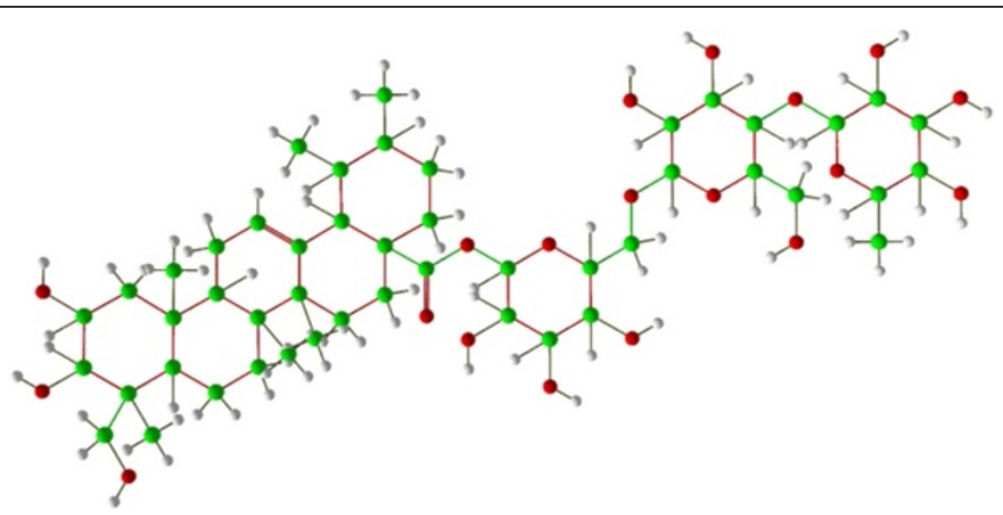

Figure 2 All-atoms molecular structure of asiaticoside (PubChem: CID 108062). 


\section{Molegro Virtual Docker}

MVD is an automated docking software program with fast processing that automatically adds the missing hydrogen atoms of the ligand and receptor molecules, if any. The software also has a module to create a surface over the receptor molecule and identify potential binding sites for its activity. The program gives 10 conformational positions or so-called poses for the ligand and returns the five best poses with MoleDockScore (equivalent to energy of binding/docking energy) and other thermodynamically calculated values. The MoleDockScore is an anonymous value by which one can suggest the best-docked ligand with its conformation. MVD also presents hydrogen bond information together with other thermodynamic values that suggest the formation of stable complexes between ligands and receptor molecules. MVD performs flexible ligand docking with optimization of the ligand geometry during docking, thus indicating bond angles, bond lengths and torsional angles of the ligand are modified at the stages of receptorligand complex generation.

\section{PatchDock}

PatchDock is a shape complementarity/geometry-based molecular docking algorithm. It is aimed at finding docking transformations that yield good molecular shape complementarity. Such transformations induce both wide interface areas and small amounts of steric clashes. A wide interface is ensured to include several matched local features of the docked molecules that have complementary characteristics. The ouput of PatchDock is a list of candidate complexes between receptor and ligand molecule. The list is sorted according to geometric shape complementarity score, approximate interface area of the receptor-ligand complex; atomic contact energy (ACE) between ligand and receptor, and 3D transformation. Finally, the server provides an option to download the ligand-receptor complexes in the PDB format.

\section{Statistical analysis}

Results were expressed as the mean \pm S.E. (standard error of mean). For intergroup differences, the data were analyzed by unpaired student's $t$-test and one-way analysis of variance (ANOVA: for more than 2 groups). ANOVA was followed by Fisher's Protected Least Significant Difference (PLSD) for post hoc comparisons. Kinetic data of nucleation-dependent fibrillation were subjected to non-linear variable-slope sigmoidal equation. The statistical program used was StatView ${ }^{\circ} 4.01$ (MindVision Software, Abacus Concepts, Inc., Berkeley, CA, USA) and GRAPHPAD PRISM (version 4.00; GraphPad Software Inc., San Diego, CA, USA). A level of $\mathrm{P}<0.05$ was considered statistically significant.

\section{Results}

Effect of asiaticoside on the diffusion time of $A \beta_{1-42}$ The diffusion time of the reference TAMRA dye was $100 \pm 5 \mu \mathrm{s}$ and that of the TAMRA-A $\beta_{1-42}$ alone was $150 \pm 5 \mu \mathrm{s}$. The autocorrelation function of TAMRA$A \beta_{1-42}$ in the (TAMRA-A $\beta_{1-42}+$ unlabeled $A \beta_{1-42}$ ) sample was best fitted with a one-component analysis model, which resulted in a diffusion time of $208 \pm 4 \mu \mathrm{s}$ (Figure 3A). However, the diffusion time of TAMRA$A \beta_{1-42}$ in the (TAMRA-A $\beta_{1-42}+$ unlabeled $A \beta_{1-42}+20$ $\mu \mathrm{M}$ of asiaticoside) samples was reduced to $164 \pm 8.0 \mu \mathrm{s}$ (Figure 3B).

\section{Effect of asiaticoside on steady-state fibrillation of $A \beta_{1-42}$} The fibrillation of $A \beta_{1-42}$ only, as measured by the steady-state ThT fluorescence intensity, was set as $100 \%$ (control). Asiaticoside at 5, 10, and $20 \mu \mathrm{M}$ significantly $(0<0.05)$ decreased fibril formation by 20,29 , and $33 \%$, respectively, compared with that of the control (Figure 4A). After examining the inhibitory effect of the three concentrations of asiaticoside on $A \beta_{1-42}$ fibrillogenesis, we also studied the effect of $20 \mu \mathrm{M}$ asiaticoside on the kinetics of $\mathrm{A} \beta_{1-42}$ fibrillation.

\section{Effects of asiaticoside on the kinetics of $A \beta_{1-42}$ fibrillation} Fibrillation kinetics of $A \beta_{1-42}$ at $50 \mu \mathrm{M}$ of concentration (the concentration used for steady-state ThT assay) displayed a very short lag phase $(<10 \mathrm{~min})$ at our experimental condition (data not shown)). This led us to assess the effect of asiaticoside on the kinetics of $A \beta_{1-42}$ fibrillation at 10 $\mu \mathrm{M}$ of concentration. At this low concentration, fibrillation appeared to be highly cooperative, reminiscent of first order phase transitions and the fibrillation kinetics exhibited characteristics of a typical nucleation and growth process (Figure 4B). The time course of fibrillation included a lag phase followed by a rapid exponential growth (elongation) of fibrils. Experimental data were fitted well by such a variable-slope sigmoidal equation curve: $[\mathrm{Y}=$ Bottom + (Top - Bottom) $/\left(1+10^{\wedge}\left(\left(\log \mathrm{T}_{50}-\mathrm{X}\right)^{*}\right.\right.$ HillSlope $\left.)\right)$; where, Bottom is the $\mathrm{Y}$ value at the bottom plateau, Top is the $\mathrm{Y}$ value at the top plateau, $\log T_{50}$ is the $\mathrm{X}$ value when the ThT fluorescence is halfway between Bottom and Top, HillSlope describes the steepness of the curve]. Lag time (LT) was defined as the time at which the tangent at the point of the maximum fibrillation rate intersects the abscissa. At $95 \%$ confidence interval, $\mathrm{T}_{50}$ (half-life) was increased by asiaticoside $\left(\mathrm{T}_{50}: 10 \mu \mathrm{M}\right.$ of $\mathrm{A} \beta_{1-42}$ vs. $10 \mu \mathrm{M}$ of $\mathrm{A} \beta_{1-42}+$ asiaticoside $=17.9 \pm 0.07$ vs. $20.5 \pm 0.13 \mathrm{~h}$; with a corresponding LT of 13.4 and $15.4 \mathrm{~h}$, respectively. Asiaticoside lengthened the time both at the lag phase and during the growth of fibrillation for $2-3 \mathrm{~h}$. 


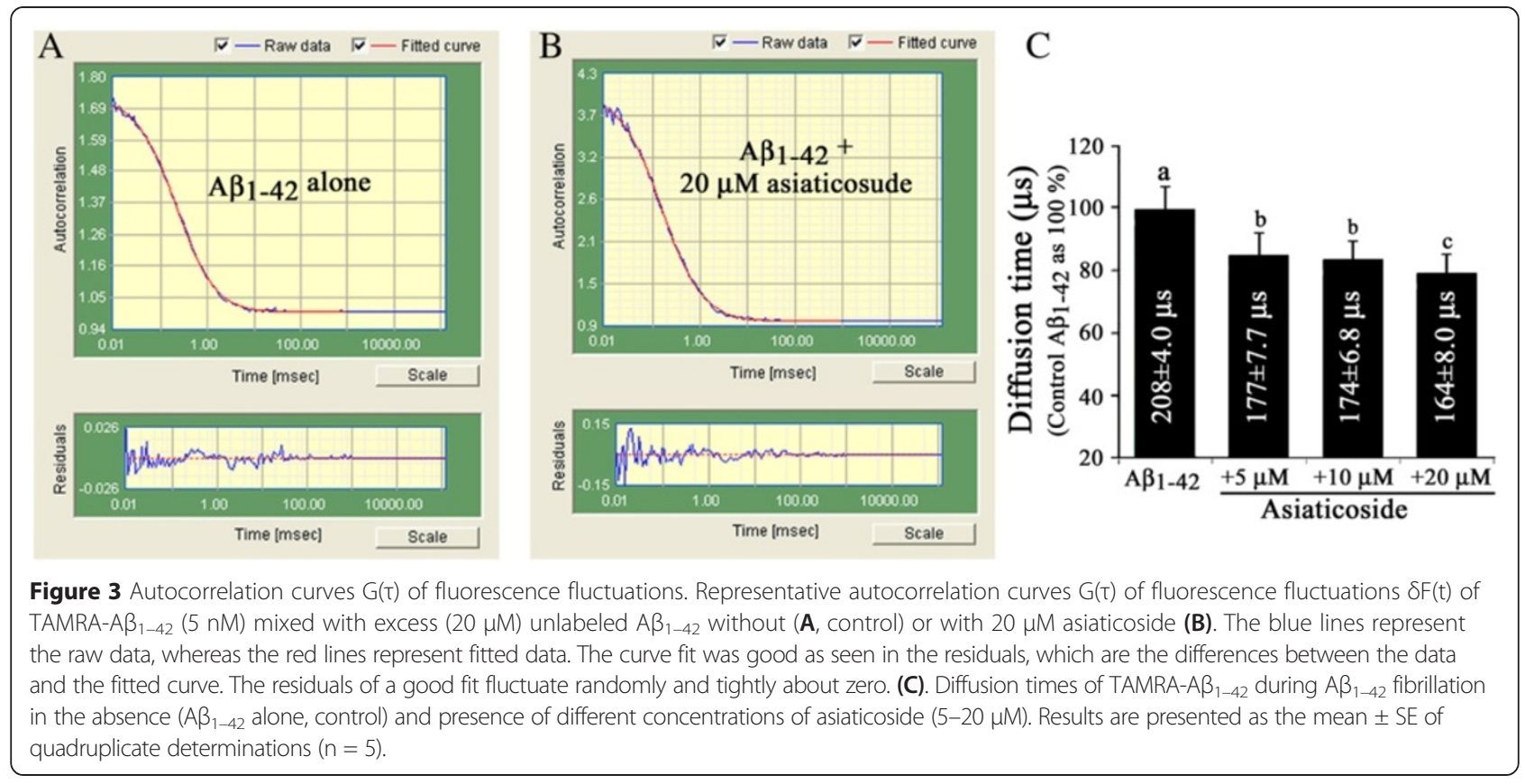

\section{Laser Scanning Microscopy (LSM)}

The fibrillations of unlabeled $A \beta_{1-42}$ and asiaticoside + unlabeled $A \beta_{1-42}$ samples also were examined by ThT laser scanning fluoromicroscopy (LSM) (Figure 5A, B), which gave green fluorescence. The fibrillations of TAMRA-A $\beta_{1-42}+$ unlabeled $A \beta_{1-42}$ and asiaticoside + TAMRA-A $\beta_{1-42}+$ unlabeled $A \beta_{1-42}$ samples were also examined by laser-scanning microscopy (LSM). TAMRA-A $\beta_{1-42}$ evoked characteristic orange fluorescence (Figure $5 \mathrm{C}, \mathrm{D}$ ). The results disclosed the precipitation of large aggregates on the glass slides. In addition, the images were processed by ImageJ to calculate the areas of the green or red fluorescence $A \beta$-aggregates.
Area $<5.0 \mu \mathrm{M}^{2}$ was not included in the image processing. The $A \beta_{1-42}+$ asiaticcoside samples had a lower number of green-fluorescent areas than did the $\mathrm{A} \beta_{1-42}$ untreated controls. Total green fluorescent areas were $768.7 \pm 9.9$ and $92.1 \pm 3.4 \mu \mathrm{M}^{2}$, respectively in the controls and asiaticcoside-treated samples (Figure 5A, B), suggesting that the aggregations of $\mathrm{A} \beta \mathrm{s}$ were inhibited by asiaticcoside. The red spots (Figure $5 \mathrm{C}, \mathrm{D}$ ) are amyloid deposits of labeled amyloid $A \beta_{1-42}$ (TAMRA-A $\beta_{1-42}$ ). Again, the number of red spot areas was significantly lesser in the presence of asiaticoside, indicating asiaticoside inhibited the fibrillation and consequently the amyloid deposits. Total areas of red-fluorescence areas were, respectively,

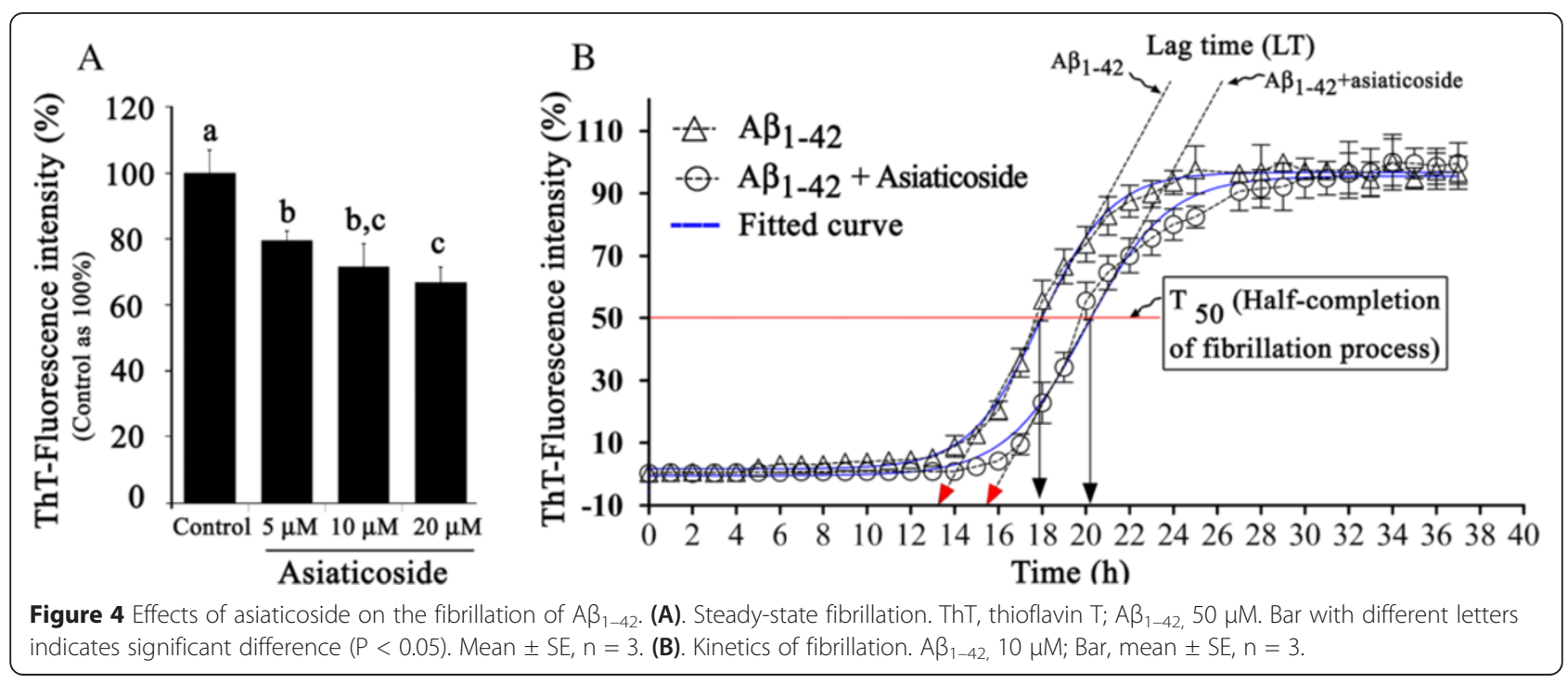



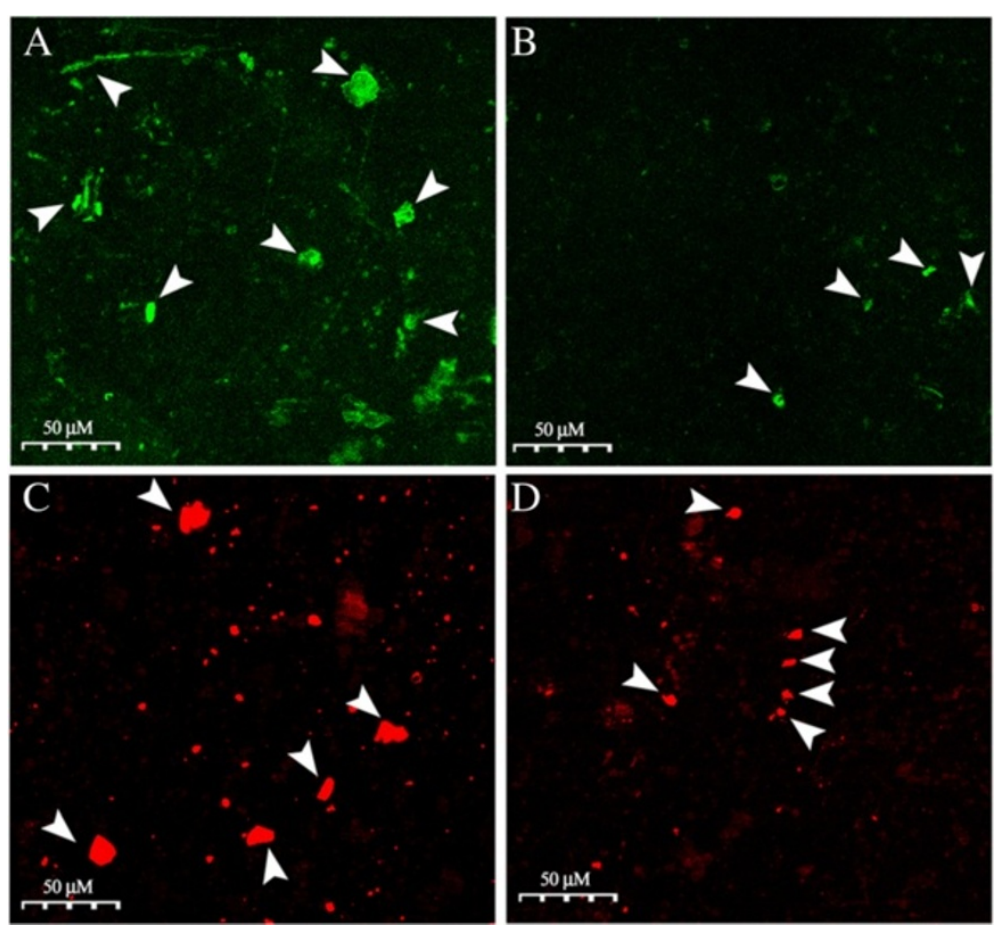

Figure 5 Visualization of amyloid aggregates by laser-scanning microscopy (LSM). Images were taken after overnight of aggregation at $37^{\circ} \mathrm{C}$ with a concentration of $50 \mu \mathrm{M}$ unlabeled $A \beta_{1-42}(\mathbf{A})$ and $5 \mathrm{nM}$ TAMRA-A $\beta_{1-42}+20 \mu \mathrm{M}$ unlabeled $A \beta_{1-42}$ (C). Aggregates of (50 $\mu \mathrm{M}$ unlabeled) $A \beta_{1-42}$ displayed characteristic green fluorescence after their binding with ThT. TAMRA-A $\beta_{1-42}$ itself exhibited orange fluorescence. Asiaticoside-incubated samples had a lower number and smaller green (ThT) (B) and orange (TAMRA-A $\beta_{1-42}$ ( $(\mathbf{D})$ fluorescent areas than those of the controls, suggesting that $A \beta_{1-42}$ fibrillation was inhibited by asiaticoside.

$2513 \pm 9.4$ and $481 \pm 18.7 \mu \mathrm{M}^{2}$ for the control and asiaticoside-treated samples.

\section{Effect of asiaticoside on $A \beta_{1-42}$ morphology}

The control samples (A $\beta_{1-42}$ alone) contained abundantly aggregated amyloid fibrils in the assembly buffer, whereas the $A \beta_{1-42}+$ asiaticoside samples contained only very small amounts of aggregates. The former fibrils exhibited a typical filamentous and branching morphology (Figure 6A and its inset). The lengths of the fibers differed from grid to grid, and the fibers were nearly inconspicuous because of the presence of extensive branching. The fibers of the $A \beta_{1-42}+$ asiaticoside samples (Figure 6B) displayed spaced, beaded and spheroidal structures, leading to necklace-like diffused proto-fibrillar filaments. The protofibrillar structures were found to align along the long axis of the fibers as if, they were 'on pathway' to complete the long fibers (arrow head). In the presence of $20 \mu \mathrm{M}$ asiaticoside, these fibers exhibited as more diffused, oligomeric and cluster-like structures.

\section{Salient features of $\mathrm{A} 1$ monomers and $\mathrm{A} 1-\mathrm{B} 1$ dimers}

The ANCHOR server (http://iupred.enzim.hu/) identified amino acids 1-18 that remained in a generally disordered region. These amino acids do not adopt a stable structure and cannot engage in sufficient numbers of favorable intrachain interactions (Figure 7A). The A1 monomer split from 2BEG is shown in Figure 7B, while the A1-B1 dimer generated by RosettaDock is shown in Figure 7C. The RosettaDock server gave the 10 bestscoring structures in rank order by energy after 1000 independent simulations as well as a plot of the energies of all 1000 structures created. Each point on the plot represents a structure created by the server (Figure 7D). The $\mathrm{x}$-axis is a distance from the starting position, and the $y$-axis is the score (energy) of the structure. A hallmark of a successful run is an energetic "funnel" of low-energy structures clustered around a single position. Lührs et al. [24] also reported that residues 1-17 of the $\mathrm{A} \beta_{1-42}$ are disordered; however, residues 18-42 form a beta strand-turn-beta strand motif that contains two parallel, in-register beta sheets that are formed by residues $18-26(\beta 1)$ and $31-42(\beta 2)$. Intermolecular side chain contacts are formed between the odd-numbered residues of strand $\beta 1$ of the $n$th molecule and the even-numbered residues of strand $\beta 2$ of the $(n-1)^{\text {th }}$ molecule. This interaction pattern leads to partially unpaired $\beta$-strands at the fibrillar ends, which explains the sequence selectivity, cooperativity, and apparent unidirectionality of A $\beta$ fibril growth [24]. 


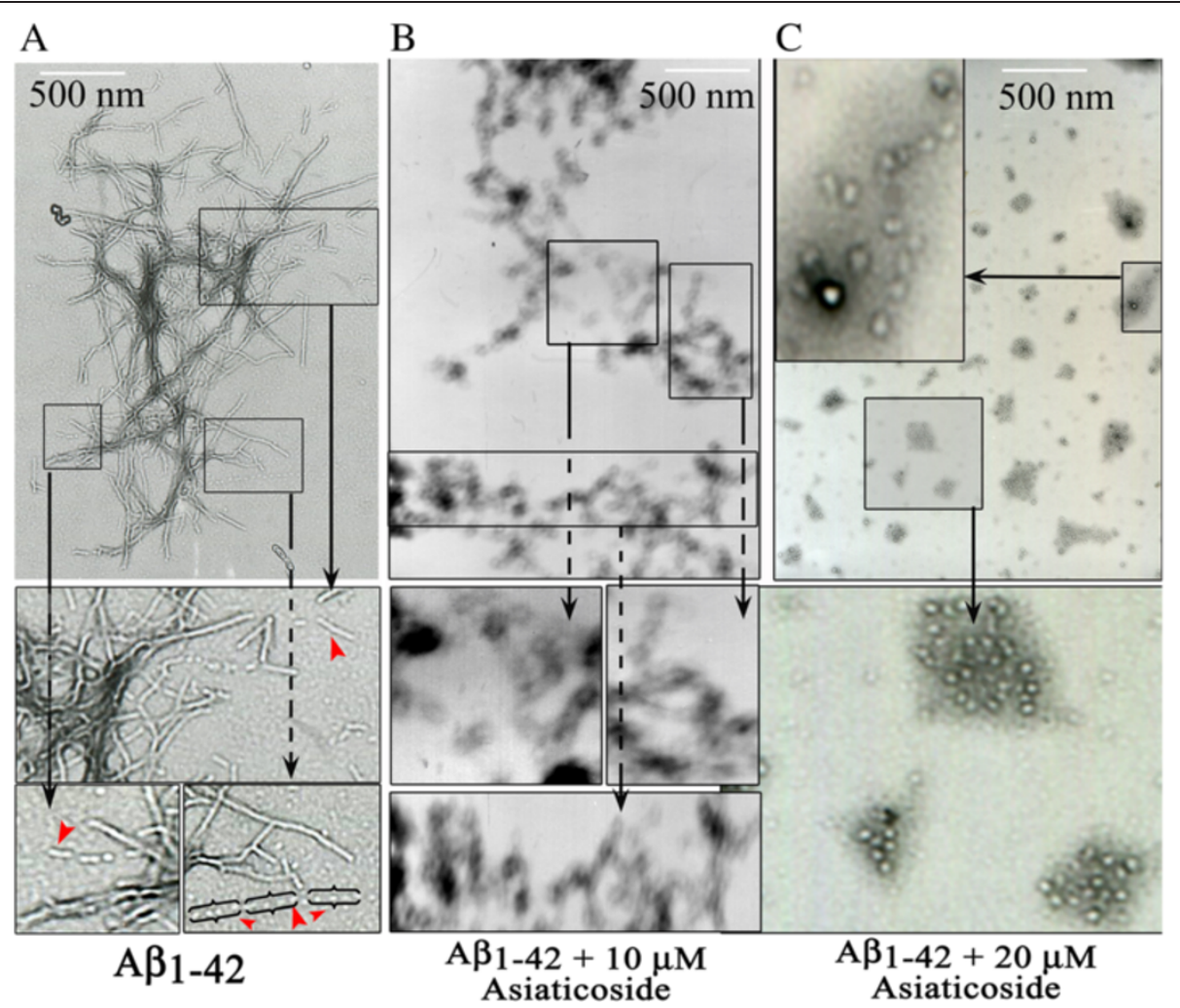

Figure 6 Representative transmission electron microscopic (TEM) views of the effects of asiaticoside on fibrillation. $\mathbf{A}$ : Control (A $\beta_{1-42}$ alone), Insets of A show complete fibers, protofibrills, and oligomeric globular amyloid species appeared to arrange in lines (red arrow) to form long fibers; $\mathbf{B}: A \beta_{1-42}+10 \mu \mathrm{M}$ asiaticoside; and $\mathbf{C}: A \beta_{1-42}+20 \mu \mathrm{M}$ asiaticoside. The fibers (B, C) became unstructured, diffused, and irregular, sometimes they appeared as globular structures (insets of $\mathbf{B}$ and $\mathbf{C}$ ). The globular structures appeared more dispersed at $20 \mu \mathrm{M}$ of concentrations of asiaticoside than those at $10 \mu \mathrm{M}$. These results indicate that asiaticoside clearly inhibited $A \beta_{1-42}$ fibrillation.

\section{Binding sites/pocketness of the monomer and dimer}

The binding and active sites of proteins are often associated with structural pockets and cavities. The results of analyses of pocketness of the monomer and dimer are shown in Figure $7 \mathrm{E}$ and $\mathrm{G}$, respectively. In the A1 monomer, the cluster with the highest degree of pocketness was located between residues $17-20$ and 35-42 (Figure 7F). The degree of pocketness was higher in the A1-B1 dimer (Figure 7H). The Q-site finder also identified pocketness in similar regions of the monomers and dimer (data not shown).

\section{Aggregation-prone amino acid residues of $A \beta_{1-42}$}

The hotspots for aggregation are shown in Figure 8. The FoldAmylod analysis revealed that residues 17-21 and 33-36 of $A \beta_{1-42}$ were aggregation-prone, whereas the AGGRESCAN analysis identified residues 17-22 and 29-42 as the aggregation-prone regions. Residues 17-20 and 35-42 of $A \beta_{1-42}$ also exhibited a propensity for aggregation upon cross-examination using the ProA server.

\section{Monomer-monomer and dimer intersurface interaction sites}

The amino acid residues involved in intersurface interactions are also shown in Figure 8. The intersurface interaction sites of the A1-B1 dimer included Phe19, Phe20, Met35, Val36, Gly37, Gly38, and Val40 of the A1 monomer and Val18, Phe19, Phe20, Ile32, Gly33, Leu34, Val36, Gly37, Val39, and Ile41 of the B1 monomer. The intersurface analysis also revealed that amino acids Gly25, Gly29, and Gly38 of B1 monomer remained buried in the interior of the dimer. The intersurface interaction sites were also rechecked using the KFC2 server, and the sites were located on both the A1 and B1 monomers (Figure 8).

\section{Docking results}

Molegro Virtual Docker (MVD)

Docking of asiaticoside with the monomers generated five poses with unique chemical arrangements. In MVD, the best pose is assessed on the basis of scoring function (MolDock score). The score ( $\mathrm{Kcal} / \mathrm{mol}$ ) mimics the potential energy change, when the protein and ligand 


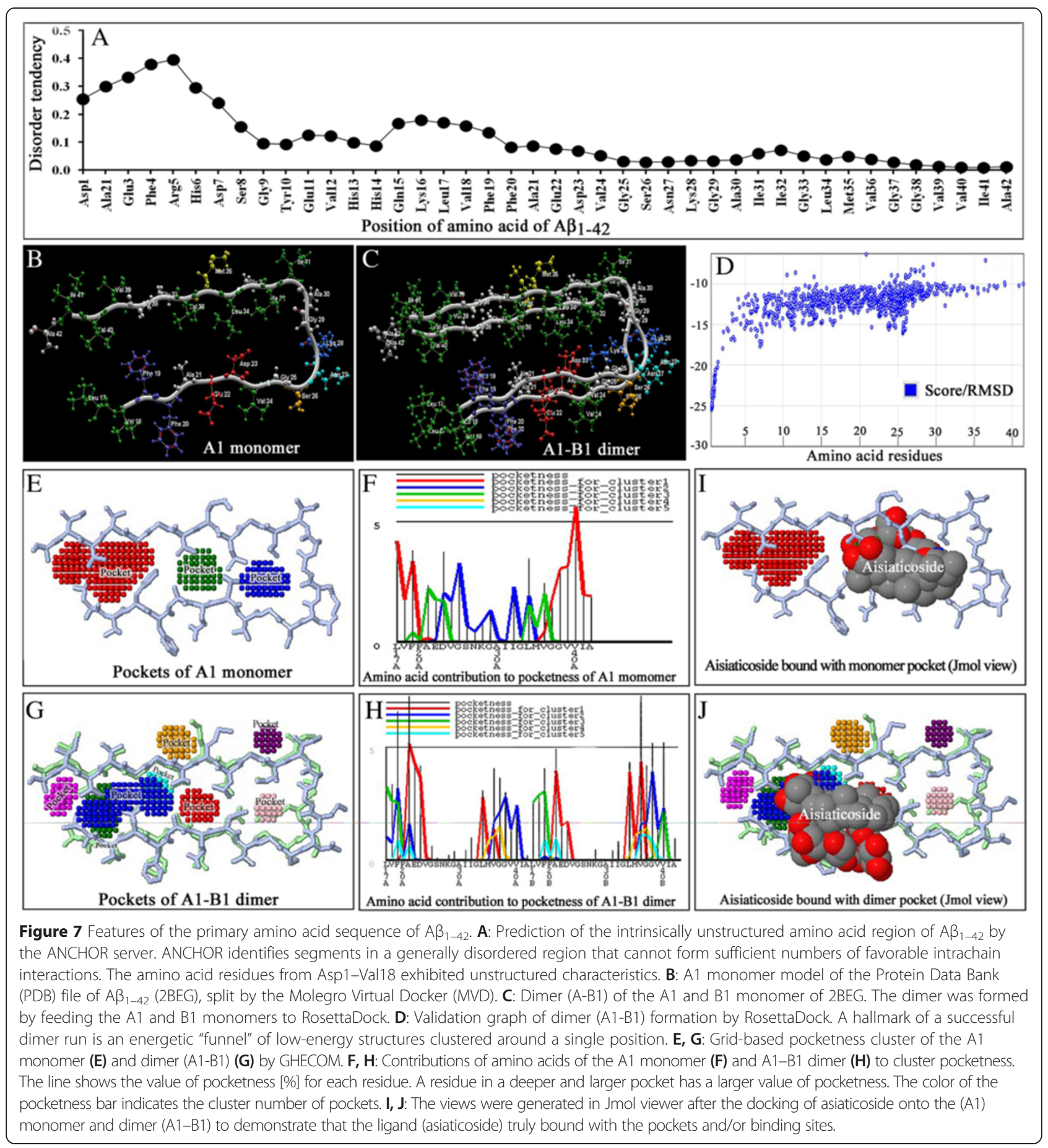

come together. This means that a very negative score corresponds to a strong binding and a less negative or even positive score corresponds to a weak or non-existing binding. Water molecules were not included in the docking setup, thus the 'water-ligand interaction' did not contribute to the MolDock score. The MolDock score of pose 1 was lower than that of the other four poses (pose $1=-117.85$, pose $2=-117.10$, pose $3=-107.27$, pose $4=-105.83$, and pose $5=-104.92 \mathrm{Kcal} / \mathrm{mol}$ ), indicating the structure in pose 1 was superior. The MolDock scores of the five poses for docking with the dimer were as follows: pose $1=-134.48$, pose $2=-118.74$, pose $3=-118.64$, pose $4=-113.89$, and pose $5=-109.09 \mathrm{Kcal} / \mathrm{mol}$. The results of the computational docking of asiaticoside with the A1monomer and A1-B1dimer are shown in Figure 9. Figure 9A presents the findings for pose 1 


\begin{tabular}{|c|c|c|c|c|c|c|c|c|c|c|c|c|c|c|c|c|c|c|c|c|c|c|c|c|c|c|c|c|}
\hline \multicolumn{3}{|c|}{ Sequence of amino acid in $A \beta 1-42$} & Leu & Val & Phe & Phe & Ala & Glu & Asp & Val & Gly & Ser & Asn & Lys & Gly & Ala & Ile & Ile & Gly & Leu & Met & Val & Gly & Gly & Val & $\mathrm{Val}$ & Ile & Ala \\
\hline \multicolumn{3}{|c|}{1} & 17 & 18 & 19 & 20 & 21 & 22 & 23 & 24 & 25 & 26 & 27 & 28 & 29 & 30 & 31 & 32 & 33 & 34 & 35 & 36 & 37 & 38 & 39 & 40 & 41 & 42 \\
\hline \multirow{3}{*}{$\begin{array}{c}\text { Aggregation-pron } \\
\text { amino } \\
\text { acids }\end{array}$} & \multicolumn{2}{|l|}{ FolAmyloid $^{1}$} & & & & & & & & & & & & & & & & & & & & & & & & & & \\
\hline & \multicolumn{2}{|l|}{ AGGRESCAN $^{2}$} & & & & & & & & & & & & & & & & & & & & & & & & & & \\
\hline & \multicolumn{2}{|l|}{$\operatorname{ProA}^{3}$} & & & & & & & & & & & & & & & & & & & & & & & & & & \\
\hline \multirow{4}{*}{$\begin{array}{c}\text { Monomer-monom } \\
\text { interaction } \\
\text { sites }\end{array}$} & \multirow{2}{*}{ Cons-PPISP ${ }^{4}$} & $\mathrm{~A} 1$ & & & & & & & & & & & & & & & & & & & & & & & & & & \\
\hline & & B1 & & & & & & & & & & & & & & & & & & & & & & & & & & \\
\hline & \multirow{2}{*}{$\mathrm{KFC}^{5}$} & $\mathrm{~A} 1$ & & & & & & & & & & & & & & & & & & & & & & & & & & \\
\hline & & B1 & & & & & & & & & & & & & & & & & & & & & & & & & & \\
\hline \multirow{3}{*}{$\begin{array}{c}\text { Asiaticoside- } \\
\text { docking } \\
\text { by } \text { MVD }^{6}\end{array}$} & Monomer (A1) & A1 & & & & & & & & & & & & & & & & & & & & & & & & & & \\
\hline & \multirow{2}{*}{ Dimer (A1B1) } & $\mathrm{A} 1$ & & & & & & & & & & & & & & & & & & & & & & & & & & \\
\hline & & B1 & & & & & & & & & & & & & & & & & & & & & & & & & & \\
\hline \multirow{2}{*}{$\begin{array}{c}\text { Asiaticoside- } \\
\text { docking by } \\
\text { PatchDock }\end{array}$} & Monomer (A1) & $\mathrm{A} 1$ & & & & & & & & & & & & & & & & & & & & & & & & & & \\
\hline & Dimer (A1B1) & $\mathrm{A} 1$ & & & & & & & & & & & & & & & & & & & & & & & & & & \\
\hline
\end{tabular}

Figure 8 Analyses of aggregation-prone amino acid residues of AB1-42 peptide chain and monomer(A1)-monomer(B1) (A1B1dimer) interaction-sites, and interaction-sites between asiaticoside vs. monomer (A1) and dimer (A1B1) after docking. ${ }^{1}$ FoldAmyloid describes a method of prediction of amyloidogenic regions from protein sequence [27], while ${ }^{2}$ AGGRESCAN predicts and evaluates of "hot spots" of aggregation in polypeptides. By identifying aggregation-prone segments in proteins, AGGRESCAN facilitates the identification of possible therapeutic targets for anti-depositional strategies in conformational diseases [28]. ${ }^{3}$ ProA: Protein Aggregation Prediction Server, which provides access to two protein aggregation propensity prediction algorithms, based on the amino acid physicochemical properties important to protein aggregation [29]. ${ }^{4}$ Cons-PPISP is a consensus neural network method for predicting protein-protein interaction sites. Given the structure of a protein, cons-PPISP predicts the residues that likely form the binding site for another protein. The inputs to the neural network include position-specific sequence profiles and solvent accessibilities of each residue and its spatial neighbors. The neural network is trained on known structures of protein-protein complexes [32]. ${ }^{5}$ KFC2 (Knowledge-based FADE and Contacts) server predicts binding "hot spots" within protein-protein interfaces by recognizing structural features indicative of important binding contacts. The server analyzes several chemical and physical features surrounding an interface residue and predicts the classification of the residue using a model trained on prior experimental data [33,34]. ${ }^{6,7}$ For comparison, whether aisaticoside-binding site overlaps with the aggregation-prone hot-spot amino acids and/or the monomer-monomer (A1-B1dimer) intersurface interaction sites, the docking results, derived from the Molegro Virtual Docker (MVD)6 [23] and PatchDock7 [35] of Figure 9 are also shown here.

docked onto the A1 monomer, and Figure 9C presents data for pose-1 docked onto the dimer (A1-B1). The amino acids with which asiaticoside bound when it docked onto the monomer (A1) and dimer (A1-B1) were visualized by the contact maps between the atoms of asiaticoside and the $A \beta_{1-42}$ amino acid residues using MVD (Figures 8 and 9B and 9D). Residues Ala21, Glu22, Asp23, Gly25, Lys28, Gly33, Leu34, and Met35 of the A1 monomer bound with asiaticoside (Figure 9B). The amino acid residues of the A1-B1 dimer that sterically interacted with asiaticoside were Leu17, Phe19, Phe20, Ala21, Glu22, Asp23 (from the A1 monomer), and Gly38 (from the B1 monomer) (Figure 9D). The higher free energy of binding between asiaticoside bound to the dimer than that of asiaticoside bound to the monomer suggests a stronger binding with the dimer.

\section{PatchDocker}

The PatchDock server provided a web page that presents the top 20 solutions. The best-scored receptor-ligand complex (Top 1) was visualized by Marvin Space (Figure 9E, 9G). Afterwards, the Top1 complex was subjected to Molegro Virtual Viewer (MVV) to show the atomic contact maps between receptor-ligand complex (Figure 9F, 9H). The results of the PatchDock revealed that amino acids
Asp23,Val24, Gly25, Ser26, Asn27, Lys28, Ile32, Gly333, Leu34, Met35, Val36 and Gly37 of the monomer bound (contacted) with asiaticoside (Figure 9F). While $A \beta_{1-42}$ dimer was docked with asiaticoside, the amino acid residues Leu17, Val18, Phe19, Val36, Val40, Ile41 and Ala42 of A1-monomer, and those of the Val40 and Ala42 of B1-monomer bound with asiaticoside (Figure 9H). The common features between Molegro Virtual Docker and PatchDocker were displayed in their common amino acid binding sites for asiaticoside: Asp23, Gly 25, Lys28, Gly33, Leu34 and Met35 of monomer were bound with asiaticoside in both of the docking systems. Consistently, the amino acids Leu17 and Phe19 of A1 monomer were common when the dimer (A1-B1) was docked with asiaticoside by both the Molegro Virtual Docker and PatchDocker.

\section{Discussion}

Fluorescence correlation spectroscopy (FCS) is a method based on fluorescence intensity fluctuations that result from dynamic movement and fluorescence quantum yield of fluorescent molecule while it diffuses in and out of small detection area. In this study we recorded the effect of asiaticoside on the dynamical properties of $A \beta_{1-}$ ${ }_{42}$ fibrillation in FCS. The initial steps of $A \beta$ aggregation are preceded by the transformation of $\alpha$-sheets to $\beta$ - 

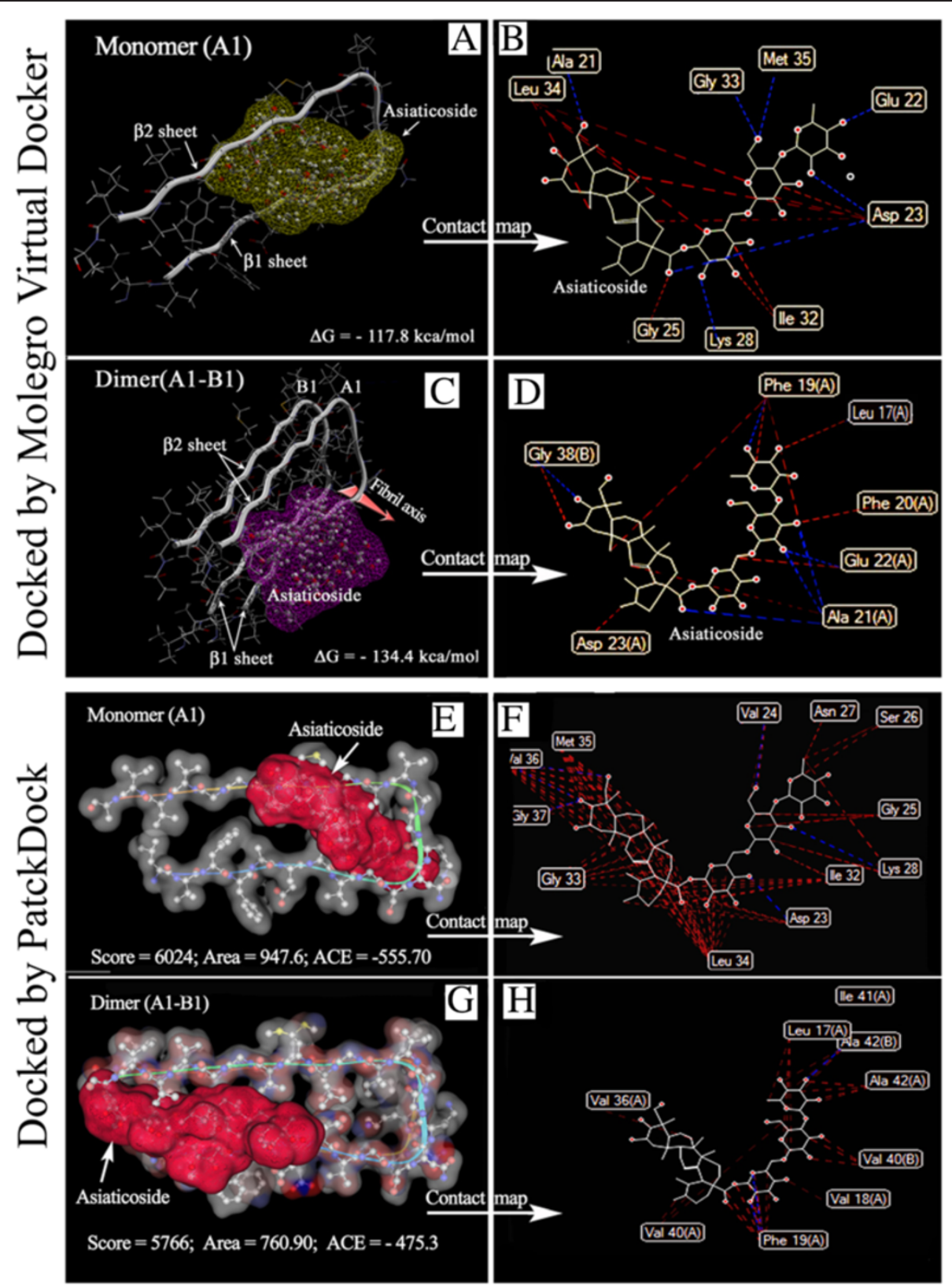

Figure 9 Docking poses of asiaticoside onto $A \beta_{1-42}$ monomer and dimer. Best docking poses of the ligand (asiaticoside) and receptor (monomer and dimer) by the Molegro Virtual Docker $(\mathbf{A}, \mathbf{B}, \mathbf{C}, \mathbf{D})$ and PatchDock $(\mathbf{E}, \mathbf{F}, \mathbf{G}, \mathbf{H})$. $\mathbf{A}, \mathbf{C}$ : Snapshot of asiaticoside in the best pose 1 docked onto the A1 monomer and A1-B1 dimer. The interaction (binding) energy $(-\Delta G)$, as the MolDock score, is shown in the figures. PatchDock $(E, F, G, H)$ : Snapshot of the Patch docking of asiaticoside on to monomer $\mathbf{( E )}$ and dimer $\mathbf{( G )}$ on the geometry-based molecular docking algorithm. The best-scored complex is shown here by the Marvin molecular viewer. Score: Geometric shape complementarity score. Area: Approximate interface area of the complex (receptor-ligand), ACE: Atomic contact energy. Atomic contact maps the receptor-ligand ( $A \beta_{1-42}$-asiaticoside) complexes (B, D of Molegro docker; $F, H$ of PatchDocker) and hydrogen maps were delineated by feeding them to Molecular Viewer. Red line indicates hydrophobic interactions, Blue line indicates hydrogen bonds.

sheets, through lateral stacking of dimers, trimers, tetramers, and oligomers, leading to matured amyloid fibers [21]. Autocorrelation function of FCS analysis revealed a diffusion time of $100 \pm 5 \mu \mathrm{s}$ for the reference dye TAMRA, vs. $150 \pm 5 \mu$ s for TAMRA-A $\beta_{1-42}$ alone. The increased diffusion time of the latter is consistent with its increased molecular mass. At time zero, the diffusion time of TAMRA-A $\beta_{1-42}$ was not significantly different in the presence or absence of asiaticoside, demonstrating that the amyloids were in their monomeric state at the start and/or early steps of fibrillation. After $1 \mathrm{~h}$ of incubation, the diffusion time of TAMRA-A $\beta_{1-42}$ in the 
TAMRA-A $\beta_{1-42}+$ unlabeled $A \beta_{1-42}$ samples increased to $208 \pm 4 \mu \mathrm{s}$, indicating that amyloid species/units with a molecular mass relatively greater than the previous one occurred in the early steps, including oligomrizations of $A \beta$ fibrillation process. This resulted in a concurrent slowness of movements of the amyloid units during "entering-andleaving" the confocal volume of FCS. In FCS, larger amyloid aggregates (of $A \beta_{1-42}$ ) in solution diffuse less frequently through the confocal volume, thereby resulting in longer diffusion times. Thus, the decreased diffusion time in the presence of asiaticoside reflects an inhibition of $A \beta_{1-42}$ aggregation by this glycoside, allowing more $A \beta_{1-42}$ in the confocal volume to freely diffuse in and out of the confocal volume. Repeated FCS measurements of 10-s sampling times illustrated an increase in the amplitude ( $y$-axis). The amplitude reflects the inverse of the average number of fluorescent TAMRA-A $\beta_{1-42}$ molecules in the focal volume. In other words, the correlation amplitude increases in size as the intensity fluctuation grows larger. This again demonstrates that asiaticoside inhibited amyloid fibrillation, allowing an increasing number of free TAMRA-A $\beta_{1-42}$ molecules to freely diffuse in the confocal volume. The diffusion time decreased as the asiaticoside concentration increased (Figure 3C).

Recently, C. asiatica extract, which contains two major triterpene glycosides (asiaticoside and madecassoside) as active components, is increasingly being used to enhance memory [9], which is severely impaired in patients with AD. Very recently, we have reported that the oral administration of madecassoside improves the memory loss of $\mathrm{AD}$ model rats, with concurrent inhibition of $A \beta_{1-42}$ fibrillation [36]. In the present study, asiaticoside inhibited $A \beta_{1-42}$ fibrillation at the early stages of the process, hinting at the basis of the neuroprotective effects of $C$. asiatica extract and/or its active components (asiaticoside and/or madecassoside) against $\mathrm{A} \beta_{1-42}$-induced $\mathrm{AD}$.

Steady-state ThT fluorospectroscopy also revealed that asiaticoside displayed the strongest inhibition of fibrillation at $20 \mu \mathrm{M}$. The results evolved from steady-state ThT fluorospectroscopy, however, did not directly disclose about the stage(s) at which asiaticoside exerted the inhibitory effect on fibrillation. Therefore, we evaluated the effect of asiaticoside on the kinetics of $A \beta_{1-42}$ fibrillation. The ThT growth curve for $A \beta_{1-42}$ was sigmoidal showing a dependence on time, which suggests that the amyloid assembly process is cooperative. The presence of asiaticoside led to a significant increase in the lag phase of $A \beta_{1-42}$ fibrillation with a concurrent deceleration of the exponential growth of fibrils. Thus it would appear that the asiaticoside acted to reduce the rate of nucleation, and, however, once critical nuclei were formed elongation was also affected by the presence of the asiaticoside. Once the FCS measurements and kinetic and/or steady-state ThT-fluorospectroscopic measurements proved that asiaticoside delayed/inhibited fibrillation, we also examined the effect of asiaticoside on amyloid fibrillation using Laser Scanning Microscopy (LSM). The ThT-bound amyloidal aggregates (green) were clearly visible in the confocal images. The red fluorescence areas indicate the larger aggregates of TAMRA-A $\beta_{1-42}$-intercalated fibers of $A \beta_{1-42}\left(5 n M\right.$ TAMRA-A $\beta_{1-42}$ was used in excess of unlabeled $A \beta_{1-42}$ ). Total numbers of fluorescent aggregates of $A \beta_{1-42}$ control samples were much more frequent and larger than those of the $A \beta_{1-42}+$ asiaticoside samples (Figure 5). Therefore, the LSM results are consistent with those of the FCS and steady-state/kinetics of ThT fluorospectroscopic studies, illustrating that asiaticoside exhibits a strong anti-fibrillation effect.

We directly observed the high-resolution image of the dense aggregates of matured fibers, long/short or single/ few scattered matured fibrils, typical branched fibrils, protofibrils, oligomer-like, in some instances, amorphous and other unstructured smaller aggregates of $A \beta_{1-42}$ in the grid fields of TEM (Figure 6). This suggests that fibrillation of $A \beta_{1-42}$ goes through a diverse dynamic exchange of conformational stages and includes continuous shuffling and growth, particularly, during the first stages of aggregation; $\mathrm{A} \beta_{1-42}$ can adopt, at least, a globular structure or so-called nucleating center. These globular structures form oligomers leading to protofibrils, which then associate in elongated fibers, finally to the highest ordered matured $A \beta_{1-42}$ fibers. Asiaticoside-induced interference of $A \beta_{1-42}$ fibrillation also was evident from the morphology of $A \beta_{1-42}$ fibrils in transmission electron micrographs. Consistent with the fact that pretreatment of $A \beta_{1-42}$ with asiaticoside kinetically lengthened the formation of amyloid nucleating centers and reduced the protofibrillar structures, TEM grids of the $A \beta_{1-42}+$ asiaticoside samples were also devoid of complete fibers, and only a small number of fibrils exhibited a diffused, shorter and some spheroid-type morphology. Structural characterization of these spheroidal species has remained elusive. Soluble amyloid $\beta$-oligomeric intermediates, emerging during amyloid fibril assembly, represent the main molecular species responsible for toxicity to cells and tissues [15]. The presence of these indefinable spheroidal amyloid species in TEM thus may raise the question of whether aisiticoside would confer toxicity to cells, and deteriorate Alzheimer's symptoms. However, there are reports that proteins can assemble into ordered aggregates without invoking the cross- $\beta$ motif [37]. Ehrnhoefer et al. [38] showed that the polyphenol epigallocatechine gallate redirected $A \beta_{1-42}$ into unstructured and nontoxic off-pathway oligomers. Asiaticoside-induced decreases in the formation and quantity of amyloid fibers (Figures 4, 5 and 6) are thus qualitatively consistent with these reports. Therefore we speculate that the spheroidal species of amyloids seen in the TEMs of asiaticoside $+A \beta_{1-42}$ samples are not toxic oligomer species. Otherwise, asiaticoside containing centella asistica extract couldn't have beneficial effects on 
memory [9], dementia and cognition [11], neuronal damage [39], neurite outgrowth [40] and $A \beta_{1-42}$-induced neurotoxicity in $\mathrm{AD}$ model animals $[10,41]$. Our previous results with madecassoside are also consistent with the speculation [36]. Because asiaticoside kinetically decreased the diffusion time, and inhibited ThT-determined lag and growth phase of fibrillogenesis, the morphological transformation of $A \beta_{1-42}$ may be mediated by the effects of this glycoside.

Structure-based drug design has made tremendous contributions to the fields of cancer chemotherapy and drug-resistant infections. Computational structure-based drug design may, therefore, facilitate the development of novel treatments for $\mathrm{AD}$. Therefore, the docking of asiaticoside with the $A \beta_{1-42}$ monomers and dimer was used in combination with experimental data to obtain information on the binding domain and mechanism of inhibition. The ability of $A \beta_{1-42}$ to bind to itself and to different ligands in a highly specific manner is an important feature of amyloidogenesis. Kirschner et al. [42] reported that $\beta$-sheets run parallel and $\beta$-strands run perpendicular to the fibril axis. Sheet $\beta 1$ is formed by approximately residues $18-26$, and sheet $\beta 2$ is formed by approximately residues 31-42. Thus, the corresponding residues of adjacent monomers line up along the fibril [24]. The aggregation-prone amino acids, i.e., hotspots of aggregation of $A \beta_{1-42}$, were located in the regions of residues $17-22$ and $32-42$ Figure 8, which matched to the $\beta 1$ and $\beta 2$ sheets of $2 B E G$ and interaction sites of asiaticoside, respectively. While docked with the monomer in MVD (Figure 9A), asiaticoside displayed steric interactions with Ala21, Glu22, Asp23, and Gly25 of the $\beta 1$ sheet and Lys28, Gly33, Leu34, and Met35 of the $\beta 2$ sheet, thereby indicating that the formation of $\beta$-sheets (from the native $\alpha$-helix) may have been, at least partially, perturbed by asiaticoside. PatchDocking of asiaticoside onto $A \beta_{1-42}$ also revealed that asiaticoside bound with the amino acid sites located at the $\beta 1$ and $\beta 2$ domain of the $\mathrm{A} \beta_{1-42}$ (Figure 9E-H). Remarkably, both the $\beta 1$ (residues 17-21) and $\beta 2$ (residues 31-42) sheet-forming amino acids were engaged in monomer-monomer (dimer) intersurface interactions (Figure 8). These results, therefore, suggest that the intersurface interaction sites could be used as the ultimate target sites of ligands to inhibit the interactions. We previously reported that the dimer may act as one of the seeding units of $A \beta_{1-42}$ protofibrils, leading to matured fibers [21]. This stimulated us to dock the dimer (Figure 9C, G) with asiaticoside to clarify whether the docking affects the intersurface interaction sites. As expected, asiaticoside bound with the amino acids of these sensitive regions. These results again suggest that asiaticoside impedes both the $\beta$-sheet-forming amino acid residues and binds with the amino acids that remain located in the monomer-monomer intersurface and buried in the loop region, such as Gly38 (Figure 8).

\section{Conclusions}

Studies of aggregation of $A \beta_{1-42}$ and interactions between small molecules, such as, asiaticoside and $A \beta_{1-42}$ may contribute to the development of inhibitors against Alzheimer's disease. In this study, by means of a combined approach of ThT, FCS, LSM, TEM techniques, we suggest a model of how the asiaticoside interacts with $\beta_{1-42}$. Our experimental results allow us to conclude that asiaticoside inhibits early stages of fibrillogenesis through interactions with 'nucleating' amyloid species and decelerating the growth phase. In addition, the in silico, docking of asiaticoside with $A \beta_{1-42}$ appears to be consistent with the ability of this glycoside to inhibit fibrillation by acting both at the intra- and inter- $\beta$-sheet interaction sites. Finally, the results obtained support further development of asiaticoside for clinical use in $\mathrm{A} \beta_{1-42}$-induced neurodegenerative diseases, such as Alzheimer's disease.

\section{Competing interests}

The authors declare that they have no competing interests.

\section{Authors' contributions}

$\mathrm{SH}$ conducted the analysis. $\mathrm{MH}$ conducted a critical review of the manuscript and provided final editing to the manuscript. SH and $\mathrm{MH}$ contributed to the conception and design of the study. All authors made substantial

contributions to drafting the manuscript. All authors read and approved the final manuscript.

\section{Acknowledgements}

This work was supported in part by a Grant-in-Aid for Scientific Research (C) from the Ministry of Education Culture, Sports, Science and Technology, Japan (23500955, M.H.).The authors gratefully acknowledge the supports of Koji Miwa, Ph.D. student of the Dept. of Environmental Physiology, Shimane University Faculty of Medicine, Izumo Japan, for helping in acquisition of FCS data. No conflict of interest exists.

\section{Author details}

${ }^{1}$ Department of Environmental Physiology, Shimane University Faculty of Medicine, Izumo 693-8501, Japan. '2Department of Biochemistry and Molecular Biology, Jahangirnagar University, Savar, Dhaka, Bangladesh.

Received: 31 December 2014 Accepted: 16 March 2015

Published online: 14 April 2015

\section{References}

1. Selkoe DJ. The molecular pathology of Alzheimer's disease. Neuron. 1991;6(4):487-98.

2. Seubert P, Vigo-Pelfrey C, Esch F, Lee $M$, Dovey $H$, Davis D, et al. Isolation and quantification of soluble Alzheimer's $\beta$-peptide from biological fluids. Nature. 1992;359(24 Sep):325-7.

3. Iwatsubo T, Odaka A, Suzuki N, Mizusawa H, Nukina N, Ihar Y. Visualization of $A \beta 42(43)$ and $A \beta 40$ in senile plaques with end-specific $A \beta$ monoclonals: Evidence that an initially deposited species is Aß 42(43). Neuron. 1994;13(1):45-53.

4. Diwan PC, Karwande I, Singh AK. Anti-anxiety profile of mandukparni Centella asiatica Linn in animals. Fitoterapia. 1991;62:255-7.

5. Bown D. Encyclopaedia of Herbs and their Uses. London, UK: Dorling Kindersley. p. 361-65

6. Chevallier A. The encyclopedia of medicinal plants. London, UK: Dorling Kindersley; 1996. p. 257.

7. Hashimoto M, Hossain S, Shimada T, Sugioka K, Yamasaki H, Fujii Y, et al. Docosahexaenoic acid provides protection from impairment of learning ability in Alzheimer's disease model rats. J Neurochem. 2002;81:1084-91.

8. Hashimoto M, Shahdat HM, Yamashita S, Katakura M, Tanabe Y, Fujiwara H, et al. Docosahexaenoic acid disrupts in vitro amyloid beta(1-40) fibrillation 
and concomitantly inhibits amyloid levels in cerebral cortex of Alzheimer's disease model rats. J Neurochem. 2008;107:1634-46.

9. Kapoor L. Handbook of Ayurvedic medicinal plants. Boca Raton, Fla, USA CRC Press; 1990

10. Mook-Jung I, Shin JE, Yun SH, Huh K, Koh JY, Park HK, et al. Protective effects of asiaticoside derivatives against beta-amyloid neurotoxicity. J Neurosci Res. 1999;58:417-25.

11. De Souza ND, Shah V, Desai PD, Inamdar PK, AD'Sa A, Ammonamanchi R, et al. 2, 3, 23-Trihydroxy-urs-12-ene and its Derivatives, Processes for their Preparation and their Use (1990); European Patent 383, A2.

12. Edelstein SJ, Schaad O, Changeux JP. Single binding versus single channel recordings: a new approach to study ionotropic receptors. Biochemistry. 1997:36:13755-60.

13. Haupts U, Maiti S, Schwille P, Webb WW. Dynamics of fluorescence fluctuations in green fluorescent protein observed by fluorescence correlation spectroscopy. Proc Natl Acad Sci U S A. 1998;95:13573-8.

14. Piehler J. New methodologies for measuring protein interaction in vivo and in vitro. Curr Opin Struct Biol. 2005;15:4-14.

15. Hossain S, Grande M, Ahmadkhanov G, Pramanik A. Binding of the Alzheimer amyloid beta-peptide to neuronal cell membranes by fluorescence correlation spectroscopy. Exp Mol Pathol. 2007;82:169-74.

16. Tjernberg L, Pramanik A, Björling S, Thyberg P, Thyberg J, Nordsted C, et al. Amyloid $\beta$-peptide polymerization studied by fluorescence correlation spectroscopy. Chem Biol. 1999;6:53-62.

17. Cavasotto CN, A bagyan RA. Protein flexibility in ligand docking and virtual screening to protein kinases. J Mol Biol. 2004;12:209-25.

18. Schoichet BK. Virtual screening of chemical libraries. Nature. 2004;43:862-5.

19. Koppen H. Virtual screening - what does it us? Curr Opin Drug Disc Dev. 2009:12:397-407.

20. Miwa K, Hashimoto M, Hossain S, Katakura M, Shido O. Evaluation of the inhibitory effect of docosahexaenoic acid and arachidonic acid on the initial stage of amyloid $\beta 1-42$ polymerization by fluorescence correlation spectroscopy. Adv Alzheimers Dis. 2013;2:66-72. http://dx.doi.org/10.4236/aad.2013.22009.

21. Hossain S, Hashimoto M, Katakura M, Miwa K, Shimada T, Shido O. Mechanism of docosahexaenoic acid-induced inhibition of in vitro Abeta1-42 fibrillation and Abeta1-42-induced toxicity in SH-S5Y5 cells. J Neurochem. 2009;111(2):568-79.

22. Marvin, "Marvin was used for drawing, displaying and characterizing chemical structures, substructures and reactions, Marvin 5.7, 2011; ChemAxon (http://www.chemaxon.com)"

23. Thomsen R, Christensen MH. MolDock: a new technique for high-accuracy molecular docking. J Med Chem. 2006;49(11):3315-21.

24. Lührs T, Ritter $C$, Adrian M, Riek-Loher D, Bohrmann B, Döbeli H, et al. 3D structure of Alzheimer's amyloid-beta (1-42) fibrils. Proc Natl Acad Sci U S A. 2005;102(48):17342-7.

25. Lyskov S, Gray JJ. The RosettaDock server for local protein-protein docking. Nucleic Acids Res. 2008;36(Web Server Issue):W233-8.

26. Dosztányi Z, Mészáros B, Simon I. ANCHOR: web server for predicting protein binding regions in disordered proteins. Bioinformatics. 2009;25(20):2745-6.

27. Garbuzynskiy SO, Lobanov MY, Galzitskaya OV. Fold Amyloid: a method of prediction of amyloidogenic regions from protein sequence. Bioinformatics. 2010;26(Oxford, England):326-32.

28. Conchillo-Sole O, de Groot NS, Aviles FX, Vendrell J, Daura X, Ventura S. AGGRESCAN: a server for the prediction and evaluation of "hot spots" of aggregation in polypeptides. BMC Bioinformatics. 2007;8(65):1-17.

29. Fang Y, Gao S, Ta D, Middaugh CR, Fang J. Identification of properties important to protein aggregation using feature selection. BMC Bioinformatics. 2013;14:314. doi:10.1186/1471-2105-14-314.

30. Kawabata T. Detection of multi-scale pockets on protein surfaces using mathematical morphology. Proteins. 2010;78(5):1195-221.

31. Laurie ATR, Jackson RM. Q-SiteFinder: an energy-based method for the prediction of protein-ligand binding sites. Bioinformatics. 2005;21(9):1908-16

32. Chenm H-L, Zhou H-X. Prediction of interface residues in protein-protein complexes by a consensus neural network method: test against NMR data. Proteins. 2005;61(1):21-35.

33. Darnell SJ, Page D, Mitchell JC. Automated decision-tree approach to predicting protein protein interaction hot spots. Proteins. 2007;68(4):813-23.

34. Zhu X, Mitchell JC. KFC2: a knowledge-based hot spot prediction method based on interface solvation, atomic density and plasticity features. Proteins. 2011;79(9):2671-83.

35. Duhovny D, Nussinov R, Wolfson HJ. Efficient unbound docking of rigid molecules. In: Guigó R, Gusfield D, et al., editors. Proceedings of the 2'nd
Workshop on Algorithms in Bioinformatics(WABI) Rome, Italy, Lecture Notes in Computer Science (LNCS) 2452. Berlin Heidelberg: Springer Verlag; 2002. p. 185-200

36. Mamun AA, Hashimoto M, Katakura M, Matsuzaki K, Hossain S, Arai H, et al. Neuroprotective effect of madecassoside evaluated using amyloid B1-42mediated in vitro and in vivo alzheimer's disease models. Intl J Indigenous Med Plants. 2014;47(2):1669-82.

37. Greenwald J, Riek R. Biology of Amyloid: structure, function, and regulation. Structure (Review). 2010;8(10):1244-60

38. Ehrnhoefer D, Bieschke J, Boeddrich A, Herbst M, Masino L, Lurz R, et al. EGCG redirects amyloidogenic polypeptides into unstructured off-pathway oligomers. Nat Struct Mol Biol. 2008;15:558-66.

39. Soumyanath A, Zhong YP, Gold SA, Yu X, Koop DR, Bourdette D, et al. Centella asiatica accelerates nerve regeneration upon oral administration and contains multiple active fractions increasing neurite elongation in-vitro. J Pharm Pharmacol. 2005;57(3):1221-9.

40. Wanakhachornkrai O, Pongrakhananon V, Chunhacha P, Wanasuntronwong A, Vattanajun A, Tantisira B, et al. Neuritogenic effect of standardized extract of Centella asiatica ECa233 on human neuroblastoma cells. BMC Complement Altern Med. 2013;13:204

41. Dhanasekaran M, Holcomb LA, Hitt AR, Tharakan B, Porter JW, Young KA, et al. Centella asiatica extract selectively decreases amyloid $\beta$ levels in hippocampus of Alzheimer's disease animal model. Phytother Res. 2009;23(1):14-9.

42. Kirschner DA, Abraham C, Selkoe DJ. X-ray diffraction from intraneuronal paired helical filaments and extraneuronal amyloid fibers in Alzheimer disease indicates cross-beta conformation. Proc Natl Acad Sci U S A. 1986;83(2):503-7.

\section{Submit your next manuscript to BioMed Central and take full advantage of:}

- Convenient online submission

- Thorough peer review

- No space constraints or color figure charges

- Immediate publication on acceptance

- Inclusion in PubMed, CAS, Scopus and Google Scholar

- Research which is freely available for redistribution 